\title{
Análise da produção científica internacional sobre segurança hídrica utilizando a base de dados Web of Science (2010-2021)
}

O conceito de segurança hídrica vai muito além da disponibilidade de água. Trata-se, de acordo com a ONU, do acesso sustentável à água de qualidade, em quantidade adequada à manutenção dos meios de vida, do bem-estar humano e do desenvolvimento socioeconômico; garantindo a proteção contra a poluição hídrica e dos desastres relacionados à água e, ainda, a preservação dos ecossistemas. Atualmente, milhões de pessoas ao redor do mundo convivem com a escassez hídrica, sujeitas às situações de risco impostas por um meio ambiente altamente degradado, proliferação de doenças vetorizadas por águas poluídas, entre outras adversidades. Diante da relevância que a segurança hídrica tem ganhado nos últimos anos no meio científico, esse artigo busca realizar uma análise bibliométrica dos avanços da produção sobre o tema. Para tanto, realizou-se uma pesquisa exploratório-descritiva, com abordagem qualitativa, usando como base de dados a Web of Science. O processamento dos dados se deu através do software VosViewer, por meio da exibição de redes bibliométricas que representam a produção científica internacional acerca do tema ora discutido. Desse modo, foram consideradas as publicações feitas entre $2010-2021$ cujo termo "water security" estava contido em seus títulos, sendo considerados apenas os documentos de tipo article e review. Observou-se que a temática possui um $\mathrm{H}$-index igual a 39. Os dados obtidos estão retratados nos gráficos apresentados neste estudo, incluindo os clusters de autores e palavras-chave mais recorrentes nos trabalhos abordados.

Palavras-chave: Segurança hídrica; Água; Análise bibliométrica.

\section{Research on international scientific production about water security using Web of Science database (2010-2021)}

The concept of water security goes far beyond of water availability. That means, according to UN (United Nations), the sustainable access to safe water, in adequate quantities to the maintenance of livelihoods, human well-being and socioeconomic development; assuring protection against hydric pollution, water disasters and conservation of ecosystems. Nowadays, millions of people around the world live with water scarcity, exposed to risks caused by highly degraded environment, proliferation of diseases vectorized by polluted waters, among other difficulties. Considering the importance that water security has gained in recent years, this work aims to do a bibliometric analysis of production advances on the theme. For this purpose, an exploratory-descriptive research was carried, with a qualitative approach, using the Web of Science database. Data processing was made using VosViewer software, through the elaboration of bibliometric networks that represent international scientific production on the topic. Therefore, article and review publications made between the years of 2010 2021 containing the term "water security" were considered. It was observed that the theme has an $\mathrm{H}$-index of 39 . Data obtained in this survey were portrayed in the graphics presented in this study, including the clusters of authors and most recurrent keywords in the studies surveyed.

Keywords: Water security; Water; Bibliometric analysis.

Topic: Planejamento, Gestão e Políticas Públicas Ambientais

Reviewed anonymously in the process of blind peer.

Paula Ângela Brunet Freitas (iD)

Universidade Federal de Campina Grande, Brasil

http://lattes.cnpq.br/0552716145369282

http://orcid.org/0000-0002-1508-2606

paulaangelabf@gmail.com

Sergio Murilo Santos de Araújo (id

Universidade Federal de Campina Grande, Brasil

http://lattes.cnpq.br/6693832655779279

http://orcid.org/0000-0001-9599-4383

sergiomurilosa.ufcg@gmail.com
Received: 23/04/2021

Approved: 24/05/2021
Referencing this:

FREITAS, P. A. B.; ARAÚJO, S. M. S.. Análise da produção científica internacional sobre segurança hídrica utilizando a base de dados Web of Science (2010-2021). Revista Ibero Americana de Ciências Ambientais, v.12, n.5, p.606-618, 2021. DOI: http://doi.org/10.6008/CBPC2179-6858.2021.005.0048 


\section{INTRODUÇÃO}

A garantia de acesso à água tratada e instalações sanitárias adequadas para a população são essenciais a uma infraestrutura de saneamento construída dentro dos parâmetros da sustentabilidade ambiental e direitos humanos. Desta feita, a busca pela superação de carências relacionadas ao esgotamento sanitário, ao manejo de resíduos sólidos e de águas pluviais urbanas configuram-se como atividades capazes de impactar significativamente na promoção da saúde e a qualidade de vida da população e, muito mais que isso, proporciona dignidade e inclusão social para as comunidades.

Ao longo do século passado, os países e, dentro deles, as bacias hidrográficas e as jurisdições locais tornaram-se a escala em que instituições em diferentes níveis gerenciam o desenvolvimento, riscos e compensações relacionados à água. Isso levou a melhores resultados em muitas nações, em termos de saúde, produtividade e gestão de ecossistemas, proporcionando maior potencial para gerenciar os riscos de sistemas variáveis. No entanto, os riscos relacionados à água permanecem muito altos e não gerenciados em muitas nações (GREY et al., 2013).

A mudança desse cenário está diretamente relacionada à segurança hídrica, que consiste na capacidade de uma população para salvaguardar o acesso sustentável à quantidade adequada de água de qualidade aceitável para sustentar os meios de subsistência, o bem-estar humano e desenvolvimento socioeconômico, para assegurar a proteção contra a poluição da água e desastres naturais e para preservar ecossistemas (UN-WATER, 2013). Na academia, a segurança hídrica é discutida em quatro escopos: a utilização da água proporcionando bem-estar econômico, melhorando a equidade social, caminhando em direção à sustentabilidade de longo prazo ou reduzindo riscos relacionados à água. (HOEKSTRA et al., 2018).

Dentro dessa mesma temática, cita-se a Agenda 2030 para o Desenvolvimento Sustentável, que traz em seu conteúdo o ODS 6 no intuito de "assegurar a disponibilidade e a gestão sustentável da água e saneamento para todos". O texto do documento menciona que a água está no centro do desenvolvimento sustentável e das suas três dimensões - ambiental, econômica e social. Os recursos hídricos, bem como os serviços a eles associados, sustentam os esforços de erradicação da pobreza, de crescimento econômico e da sustentabilidade ambiental. $\mathrm{O}$ acesso à água e ao saneamento importa para todos os aspectos da dignidade humana: da segurança alimentar e energética à saúde humana e ambiental. (PNUD, 2015)

Percebe-se, então, que a problemática decorrente da insegurança hídrica vai muito além da infraestrutura urbana, englobando o bem-estar da população e a saúde pública. Daí a importância de uma força tarefa a nível internacional, visando o acesso universal e seguro à àgua potável, reduzindo a vulnerabilidade da população já afetada pela crise hídrica, projetando investimentos em infraestrutura adequada e buscando reverter tal quadro até o ano de 2030.

A abordagem acerca da relação das pessoas com a água torna-se cada dia mais importante, uma vez que tal temática é capaz de interferir em diversos aspectos da qualidade de vida da população, pois se trata de um dos mais básicos direitos humanos. As situações de crise salientam os imperativos morais, bem como a urgente necessidade de decisões políticas e econômicas capazes de solucionar demandas mais 
imediatas.

Diante desse contexto, este artigo tem como objetivo analisar a produção científica internacional acerca da segurança hídrica, possibilitando uma maior compreensão das características dos trabalhos publicados. Para isso, utiliza-se de técnicas avaliativas (medidas de produtividade e métricas de impacto) e técnicas relacionais (coautoria e coocorrência), tendo como parâmetros os principais autores, áreas das publicações, periódicos mais influentes, países e idiomas.

Por intermédio do software VOSviewer (versão 1.6.16) também foram geradas as redes com os clusters de coautoria e as palavras-chave com maior relação e frequência nas publicações sobre a temática. A análise foi realizada através de conteúdo disponível na base de dados Web of Science no período de 2010 a 2021, como maneira de avaliar a produção científica relacionada ao tema na última década. Nesse sentido, a presente pesquisa se justifica pela relevância em disponibilizar uma visão abrangente do panorama atual considerando os avanços da pesquisa sobre a segurança hídrica ao redor do mundo.

O presente estudo se configura a partir desta introdução, seguida de uma seção de revisão teórica tratando conceitualmente da segurança hídrica. Em seguida, há uma terceira seção designada para descrever a metodologia empregada. Na quarta seção estão apresentados à discussão e os resultados, e finalmente, a exposição das conclusões do estudo, constando limitações e indicações de estudos futuros.

\section{REVISÃO TEÓRICA}

A insegurança hídrica, definida como falta de acesso à água ou capacidade limitada de garantir água segura, acessível e socialmente aceitável, é uma ameaça significativa à saúde e o bem-estar geral dos humanos. Ainda assim, globalmente, quatro bilhões de pessoas experimentam grande escassez de água em pelo menos um mês por ano, e meio bilhão de pessoas experimentam grave escassez de água ao longo do ano (MEKONNEN et al., 2016).

De acordo com Cosgrove et al. (2012), na elaboração de relatório sobre as conclusões da Fase 01 do Projeto de Cenários Hídricos pela UNESCO (WWAP Water Scenarios Project to 2050), os principais fatores que afetarão o futuro dos recursos hídricos globais são: crescimento populacional, crescimento econômico, mudanças nos padrões de produção e comércio, aumento da competição pela água devido ao aumento da demanda para fins domésticos, industriais e agrícolas e a forma como diferentes setores de a sociedade responderá à crescente escassez de água e poluição. Nas últimas duas décadas, o termo segurança hídrica tem sido cada vez mais usado tanto na academia quanto em debates políticos. Este cenário reflete as preocupações crescentes sobre a vulnerabilidade do sistema humano e terrestre quando confrontados com desafios relacionados à água, enquanto a atenção é dada principalmente às necessidades de água nacionais e regionais (COOK et al., 2012).

Grey et al. (2013) aduzem que o diálogo eficaz entre ciência e política tornou-se mais importante embora mais difícil - em um contexto de globalização. Questões de política de segurança hídrica, para conter e gerenciar riscos relacionados à água desde o domicílio até o nível global, precisam ser formuladas, de forma que a ciência possa reunir dados e evidências interdisciplinares para identificar soluções. Essa 
agenda deve superar problemas de incentivos até então intratáveis para cientistas, que não são recompensados por assumir riscos e por trabalhar entre as divisões disciplinares ou científicas, em um mundo onde a soberania nacional e o sigilo de dados comumente prevalecem em questões relacionadas à água.

A água conecta vários sistemas socioecológicos, econômicos e geofísicos em várias escalas e, portanto, constitui um sistema global. Isso deve ser considerado tanto em intervenções técnicas quanto em estruturas de governança, que devem ser bem executadas para fornecer segurança hídrica (BOGARDI et al.). Para Wheater et al. (2015), em face das crescentes pressões sobre o ambiente hídrico, uma nova ciência disciplinar é necessária para contribuir para uma compreensão mais profunda dos componentes da dinâmica do sistema de água e sua resposta às mudanças no ambiente. Assim como a gestão da água deve abordar uma gama de escalas - de fontes locais a bacias hidrográficas e aquíferos subterrâneos, às vezes envolvendo várias jurisdições -, o mesmo acontece com o a ciência subjacente, que precisa cruzar múltiplas escalas e "jurisdições disciplinares".

Os sistemas hídricos compreendem interações complexas de fatores físicos, sociais, econômicos e políticos, incluindo riscos e incertezas. Eles se tornam mais complexos nos cenários mais críticos, definidos por uma mistura de aridez natural, vulnerabilidade às inundações e alta variabilidade de chuva intra e interanual, sendo esta última a mais complexa. A complexidade aumenta a demanda de investimentos em informações, instituições e infraestrutura necessários para a segurança hídrica (GREY et al., 2007).

As áreas urbanas marcadas pela vulnerabilidade - como ocupações irregulares -, notadamente se mostram como locais propícios à veiculação de doenças associadas à má qualidade da água, visto que sistemas de drenagem abertos, saneamento deficitário, inundações, pobreza, manuseio de água contaminada e armazenamento inadequado são fatores capazes de expor a população a uma profusão de problemas de saúde.

Ross (2012) levanta a imperativa necessidade de reconhecer a ligação crítica entre água limpa e saneamento adequado na proteção da saúde infantil. Em muitos casos, saneamento inadequado e sistemas de esgoto a céu aberto contaminam gravemente o abastecimento de água, causando doenças e mortes. Entre as ameaças de segurança enumeradas sob o paradigma da segurança humana, são altamente relevantes para o problema infantil: ameaças à saúde, alimentação, meio ambiente, comunidade e segurança econômica. Existem muitos casos graves e potencialmente fatais riscos à saúde que surgem de água suja e sistemas de saneamento insalubres.

O desenvolvimento econômico também é um fator influente na interpretação da segurança hídrica e na percepção da urgência em lidar com as ameaças relacionadas à água. Indicativamente, no Sul Global, as preocupações com o desenvolvimento prevalecem, e a segurança da água é entendida principalmente por sua natureza para alcançar alto crescimento econômico e redução da pobreza (GREY e SADOFF, 2007).

Desde 2010, a Assembleia Geral das Nações Unidas reconheceu explicitamente a água e o saneamento como humanos direitos que são "essenciais para o pleno gozo da vida e de todos os direitos humanos". A escassez de água afeta mais de $40 \%$ da população mundial, número que deverá subir ainda 
mais como resultado da mudança do clima e da gestão inadequada dos recursos naturais (PNUD, 2015). A partir dessa conjuntura, observa-se a necessidade de buscar a segurança hídrica, que é fundamental para o desenvolvimento social dos países, através do estímulo à sustentabilidade e às práticas de boa governança, visando promover saúde e o bem-estar para presentes e futuras gerações.

A resposta da comunidade científica global ao desafio da política de segurança hídrica requer uma perspectiva dos sistemas centrados na água sobre os riscos complexos e interconectados que a sociedade enfrenta (GREY et al., 2013).

\section{METODOLOGIA}

Objetivando o mapeamento a produção científica internacional sobre o tema ora apresentado, realizou-se uma pesquisa exploratório-descritiva, desenvolvida através de análise bibliométrica. Para fins de conceituação, define-se que a bibliometria - ou análise bibliométrica - diz respeito à contagem de publicações ou citações encontradas nas bases de publicações científicas e acadêmicas, tendo sido utilizada como uma ferramenta prática para monitorar a tecnologia e avaliar atividades científicas (COATES et al, 2001).

O protocolo de realização da pesquisa foi iniciado com a escolha do seu tema central: segurança hídrica. Tal escolha se deu em função deste estar relacionado ao tema do Projeto de Dissertação de Mestrado da autora principal deste trabalho (realizado no Programa de Pós-Graduação em Engenharia e Gestão de Recursos Naturais da Universidade Federal de Campina Grande - UFCG). Dessa maneira, o estudo aqui desempenhado configura uma etapa preliminar de pesquisa e construção do referencial teórico do referido projeto.

A base de dados utilizada para este fim foi a coleção principal da Web of Science (Clarivate Analytics). A pesquisa foi realizada em 01 de março de 2021. Para o delineamento da amostra, consideraram-se os periódicos classificados nos índices: Science Citation Index Expanded (SCI-EXPANDED), Social Sciences Citation Index (SSCI) e Emerging Sources Citation Index (ESCI). O período considerado foi abrangido entre 2010-2021, de forma a alcançar um número expressivo de publicações, além de obter um panorama relevante sobre o crescimento da produção científica dentro da temática selecionada.

Pesquisaram-se publicações que contivessem "Segurança Hídrica” ("Water Security") em seus títulos, buscando publicações onde a expressão retratasse um elemento essencial do texto, excluindo-se aquelas onde só constasse em resumos ou palavras-chave. Como resultado desse procedimento, foram identificados 534 estudos. Em seguida foram realizados refinamentos, buscando aprimorar a investigação. Primeiro, mantiveram-se apenas as publicações constantes dos índices: Science Citation Index Expanded (SCI-EXPANDED), Social Sciences Citation Index (SSCl) e Emerging Sources Citation Index (ESCI), totalizando 459 artigos. Finalmente, foram considerados apenas os artigos tipo article e review e as com idioma inglês, de forma que o total de publicações selecionadas foram 388.

O script final da busca foi: "título: ("water security") Refinado por: índice do Web of Science: (wos.sci or wos.esci or wos.ssci) and tipos de documento: (article or review) and idiomas: (english) Tempo 
estipulado: 2010-2021. Índices: SCI-EXPANDED, ESCI, A\&HCI, SSCI, CPCI-SSH, CPCI-S."

No que tange às técnicas avaliativas, os dados foram captados e organizados utilizando a ferramenta Clarivate Analytics, disponível na própria Web of Science. Tal recurso é capaz de gerar gráficos descrevendo publicações por critério de áreas, ano, periódicos, organizações, países e idiomas em que foram escritas. As publicações também foram ranqueadas a partir do 'cit. Score' e classificadas de acordo com seu $\mathrm{H}$-Index para identificar quais autores e, por consequência, obras possuem maior impacto na área de estudo em avaliação. Para que fosse possível a construção de análises bibliométricas através do software VosViewer (versão 1.6.16), o resultado da pesquisa foi exportado da base de dados através da ferramenta 'salvar em outros formatos de arquivo', armazenando o 'registro completo e referências citadas' no tipo de arquivo 'texto sem formatação'.

A etapa seguinte constitui-se de avaliação relacionada ao ano de publicação, áreas de pesquisa, periódicos, países, idioma, autores e número de citação. O uso do software VosViewer possibilitou a organização de clusters baseados em "Co-authorship - Authors" (relação de força que ocorre quando, em síntese, autores participam de publicações em parceria); “Co-authorship-Organizations" (relação de força que ocorre quando, em síntese, organizações participam de publicações em parceria); "Co-authorship Countries" (relação de força que ocorre quando, em síntese, países participam de publicações em parceria) e "Co-occurrence - All Keywords" (relação de força e frequência entre palavras-chave) (VAN ECK et al., 2017).

\section{RESULTADOS E DISCUSSÃO}

A partir deste ponto, apresentam-se os resultados obtidos nas análises bibliométricas realizadas e considerações sobre os mesmos. Como indicadores bibliométricos foram utilizados: número de documentos publicados por ano; países onde os artigos foram publicados; áreas de pesquisa das publicações investigadas, periódicos onde houve publicações, artigos mais relevantes, bem como as palavras-chave mais frequentes nos artigos pesquisados. Na realização da análise pretendida, considerando o período compreendido entre 2010-2021 e os demais refinamentos já especificados na metodologia, no que tange à produção científica internacional acerca da segurança hídrica foi possível identificar 388 publicações indexadas na Web of Science, disponibilizadas até a data de consulta realizada em 01 de março de 2021

Da observação do gráfico, o que se pode notar é uma tendência crescente de publicações na temática da segurança hídrica que se iniciou em 2015. Tal fato pode estar atrelado à aprovação dos ODS na Cúpula das Nações Unidas sobre o Desenvolvimento Sustentável que aconteceu no mesmo ano, voltando o olhar da sociedade e da ciência para o debate de uma questão tão relevante e impactante para a humanidade. Verifica-se, ainda, um número mais expressivo na produção científica no ano de 2020, com 77 publicações indexadas na Web of Science.

Prosseguindo na análise dos dados processados pela ferramenta Clarivate Analytics, quanto às áreas de pesquisa das publicações investigadas, a área com mais publicações é Environmental Sciences 
Ecology (181), seguida por Water Sources (150) e Science Technology Other Topics (67). As três áreas correspondem a respectivamente $46.649 \%, 38.660 \%$ e $17.268 \%$ das 388 publicações consideradas neste estudo.

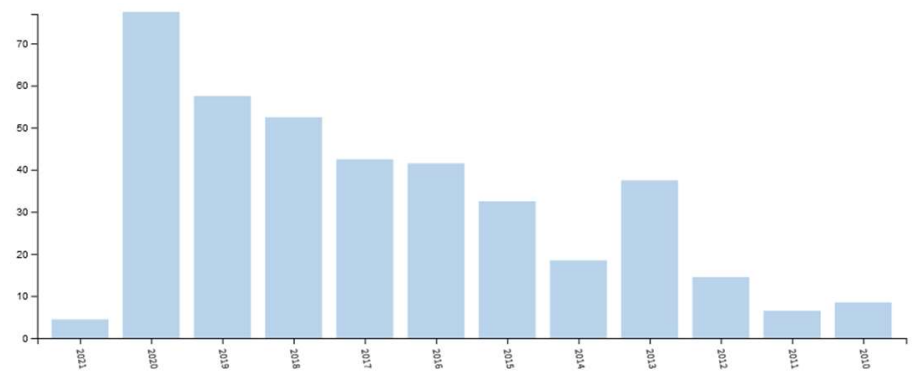

Figura 1: Quantidade de Publicações por ano (2010-2021). Fonte: Web of Science (2021).

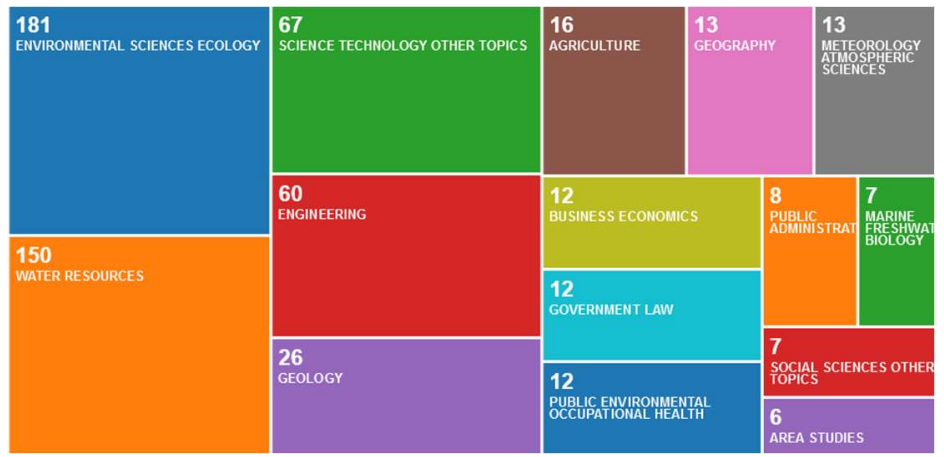

Figura 2: Campo "Áreas de pesquisa". Fonte: Web of Science (2021).

As publicações selecionadas constam em um total de 169 periódicos. Os três mais influentes são: Water, com 33 publicações (8,505\%); Current Opinion in Environmental Sustainability, com 16 publicações (4.124 \%) e Science of the Total Environment, com 12 publicações (3.093\%). Em quarto lugar, o periódico Philosophical Transactions of the Royal Society a Mathematical Physical and Engineering Sciences, com 11 publicações (2,835\%). Os periódicos Environmental Science Policy, Water International e Water Policy figuram com 10 publicações cada um, perfazendo individualmente $2.577 \%$. A partir daí, os números diminuem gradativamente até chegar a apenas uma publicação indexada.

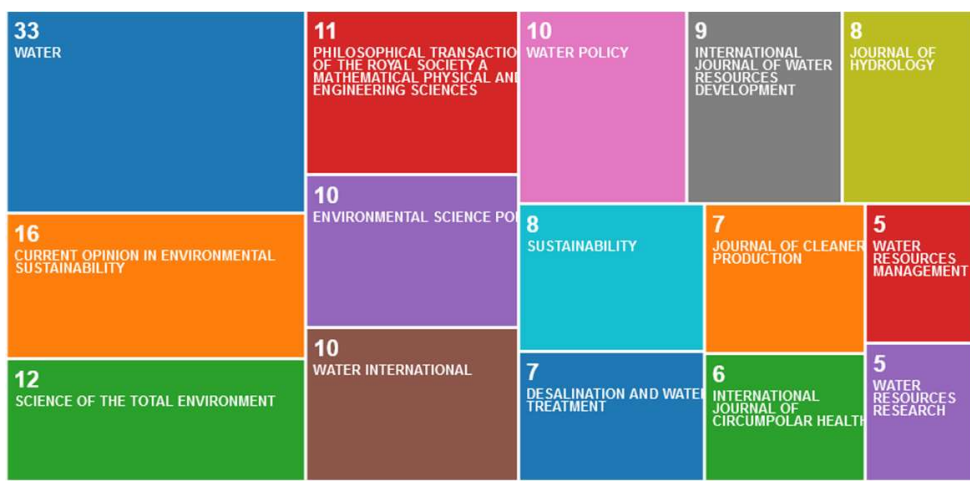

Figura 3: Campo "Títulos da Fonte". Fonte: Web of Science (2021).

Com relação à frequência de publicações por país, identificou um total de 77 países que desenvolveram algum tipo de estudo a respeito da segurança hídrica. Importante ressaltar que seis registros (1.546\%) não apresentaram dados neste campo. Relacionando os 10 países com maior número de publicações, temos: Estados Unidos (119), China (62), Canadá (58), Inglaterra (54), Austrália (30), Holanda 
(26), Alemanha (25), Índia (25), Suécia (19) e África do Sul (19). Observa-se uma vasta dominância dos Estados Unidos na produção científica sobre a temática.

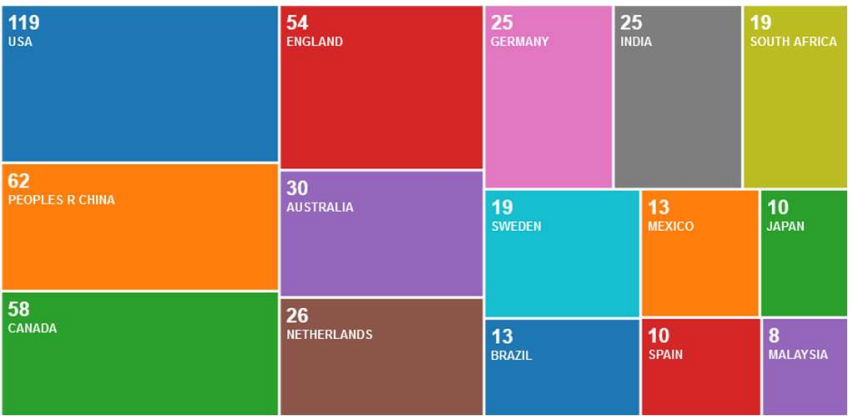

Figura 4: Campo Países e Regiões. Fonte: Web of Science (2021).

Seguindo a análise, observou-se que a temática possui um $\mathrm{H}$-index (mensurado através de ferramenta disponibilizada na própria Web of Science) igual a 39, indicando então que esse é o número de publicações com maior relevância no campo ora estudado. De acordo com esse critério, a média de citações por item é de 22.82. No quadro a seguir estão ranqueadas as 10 publicações de maior impacto utilizando-se como referência o já citado $\mathrm{H}$-index - seus autores, periódico, ano de publicação e número de citações.

Quadro 1: As dez publicações de maior impacto (H-index) constantes na Web of Science (2010-2021) sobre Segurança Hídrica.

\begin{tabular}{|c|c|c|c|c|}
\hline Título & Autores & Título da Fonte & Ano & Citações \\
\hline Global threats to human water security and river biodiversity & $\begin{array}{l}\text { Voeroesmarty } \\
\text { et al. }\end{array}$ & Nature & 2010 & 3085 \\
\hline Water security: Debating an emerging paradigm & Cook et al. & $\begin{array}{l}\text { Global Environmental Change-Human And Policy } \\
\text { Dimensions }\end{array}$ & 2012 & 291 \\
\hline $\begin{array}{l}\text { Taking the Waste Out of Wastewater for Human Water Security and } \\
\text { Ecosystem Sustainability }\end{array}$ & Grant et al. & Science & 2012 & 278 \\
\hline $\begin{array}{l}\text { Groundwater depletion during drought threatens future water } \\
\text { security of the Colorado River Basin }\end{array}$ & Castle et al. & Geophysical Research Letters & 2014 & 180 \\
\hline $\begin{array}{l}\text { Emerging opportunities for nanotechnology to enhance water } \\
\text { security }\end{array}$ & Alvarez et al. & Nature Nanotechnology & 2018 & 168 \\
\hline $\begin{array}{l}\text { Water security for a planet under pressure: interconnected } \\
\text { challenges of a changing world call for sustainable solutions }\end{array}$ & Bogardi et al. & Current Opinion In Environmental Sustainability & 2012 & 134 \\
\hline $\begin{array}{l}\text { Integrating legacy soil phosphorus into sustainable nutrient } \\
\text { management strategies for future food, bioenergy and water } \\
\text { security }\end{array}$ & Rowe et al. & Nutrient Cycling in Agroecosystems & 2016 & 104 \\
\hline $\begin{array}{l}\text { China's water security: Current status, emerging challenges and } \\
\text { future prospects }\end{array}$ & Jiang et al. & Environmental Science \& Policy & 2015 & 102 \\
\hline $\begin{array}{l}\text { Growing water scarcity in agriculture: future challenge to global } \\
\text { water security }\end{array}$ & $\begin{array}{l}\text { Falkenmark et } \\
\text { al. }\end{array}$ & $\begin{array}{l}\text { Philosophical Transactions Of The Royal Society A- } \\
\text { Mathematical Physical And Engineering Sciences }\end{array}$ & 2013 & 87 \\
\hline $\begin{array}{l}\text { Water security in one blue planet: twenty-first century policy } \\
\text { challenges for science }\end{array}$ & Grey et al. & $\begin{array}{l}\text { Philosophical Transactions Of The Royal Society A- } \\
\text { Mathematical Physical And Engineering Sciences }\end{array}$ & 2013 & 76 \\
\hline
\end{tabular}

Em rápida análise, resta bastante clara a relevância do título "Global threats to human water security and river biodiversity", escrito por Voeroesmarty et. al. e publicado no periódico Nature em 2010. O trabalho foi citado 3.085 vezes, enquanto o segundo da lista conta com 291 citações. Em suma, os autores tratam conjuntamente as perspectivas humanas e da biodiversidade sobre a segurança da água, usando uma estrutura espacial que quantifica estressores e contabiliza os impactos causados. O estudo foi capaz de quantificar que quase $80 \%$ da população mundial está exposta a altos níveis de ameaça à segurança da água, sendo os países mais pobres mais vulneráveis. O artigo conclui que as respostas de política e gestão à crise hídrica devem ser capazes de limitar as ameaças em sua origem, a fim de garantir a segurança global da água para humanos e para a biodiversidade de água doce. 
O segundo artigo mais citado foi escrito por Cook et al. (2012) e denominado "Water security: Debating an emerging paradigm". Trata-se de uma revisão abrangente do conceito de segurança hídrica que considera literaturas acadêmicas e políticas, tratando também da sua operacionalização. As autoras trazem uma comparação de definições e abordagens analíticas sobre o tema nas ciências naturais e sociais e argumentam que uma abordagem integrativa para a segurança hídrica envolve questões de boa governança. Terceiro da lista, no artigo "Taking the Waste Out of Wastewater for Human Water Security and Ecosystem Sustainability", os autores Grant et al. (2012) revisam as abordagens emergentes para reutilizar e minimizar a geração de águas residuais, de forma a aproveitar ao máximo os escassos recursos de água doce. Tais abordagens exigem mudanças na forma como a água doce é obtida, usada, gerenciada e precificada, mas atendem às diversas necessidades hídricas dos países desenvolvidos e em desenvolvimento, além de conferir uma variedade de benefícios ambientais.

A fim de robustecer a pesquisa e a demonstração gráfica de dados bibliográficos obtidos, foi empregado o software VosViewer (versão 1.6.16) para mapear as relações e clusters formados por autores de publicações que compõem o universo de dados ora em exame. Para isso, foram considerados aqueles com pelo menos uma publicação sobre segurança hídrica dentro dos parâmetros de metodologia empregados na elaboração deste trabalho. O software detectou 1296 autores, mas para uma análise mais precisa 500 foram considerados na estruturação do gráfico disposto na Figura 5.

Para compreender o gráfico representado na Figura 5, deve-se considerar que o tamanho da esfera onde está impresso o nome de cada autor é determinado pela relevância que o mesmo possui em termo de citações: nas esferas maiores estão aqueles mais citados dentro da abrangência da pesquisa realizada. Para Grácio (2016) a proximidade de dois autores identificada por sua alta frequência de cocitação pode ser originária de várias possibilidades, entre elas: compartilhamento de referencial teórico e/ou metodológico, similaridade e/ou complementaridade de conteúdos e oposição teórico e/ou metodológica, entre outras. As conexões entre as esferas representam trabalho em cooperação, expressas em coautoria de obras. E, por fim, um conglomerado de esferas com a mesma cor representa um conjunto de autores interligados entre si por relações de coautoria, denominados pelo próprio software como clusters.

No gráfico observamos o principal cluster como o de número 2, representado em verde, sendo formado por 11 autores e 36 links de coautoria. O principal deles é Varady et al. com 10 publicações e relação de força tamanho 61. Outro cluster de destaque é o 3, com 11 autores e 41 links de coautoria, representado na cor azul, cujo expoente é Scott, C. A. com 9 publicações e relação de força 52. Em seguida, temos Gerlak com 5 publicações e força tamanho 27 e Albrecht, T. R. com 3 publicações e força 23. Nele também se destacam Garffin et al. com 3 publicações cada e relação de força de 16 e 17 respectivamente. Mais um cluster de destaque é o de número 1, em vermelho, cujos 14 autores possuem em média uma relação de força 14 e no máximo três trabalhos publicados.

A Figura 6 traz a representação denominada overlay visualization de rede de coautoria por autores. Esse tipo de visualização permite uma análise da temporalidade de uso de cada termo, de acordo com a data dos documentos de onde foram extraídos, formando clusters de acordo com sua cronologia. 


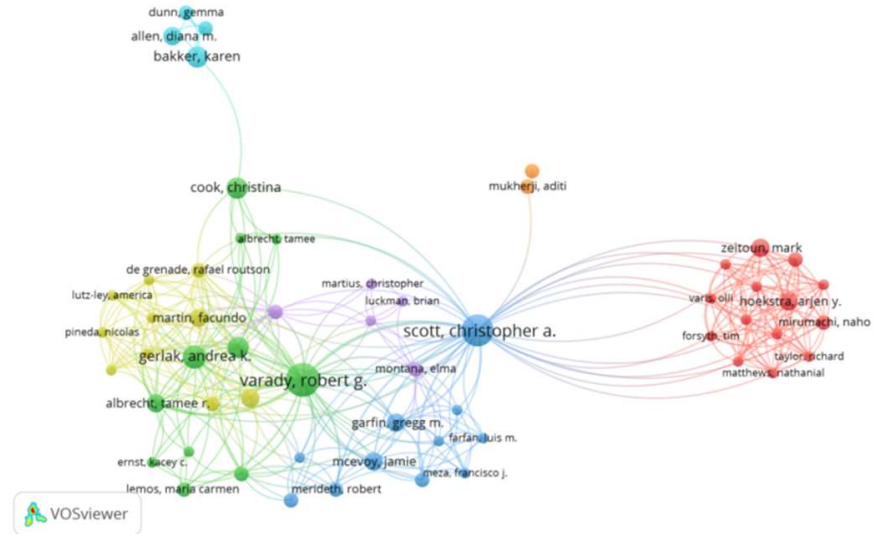

Figura 5: Rede de coautoria por autores. Fonte: Web of Science (2021).

Observa-se que, dos principais conglomerados destacados na Figura 6, as publicações mais recentes estão relacionadas principalmente nos clusters 2 e 3, capitaneados por Varady et al., que são os autores mais relevantes nesta análise. As publicações utilizadas como base são de 2016 e 2015, respectivamente. Diante disso, podemos perceber que as questões levantadas por tais autores continuam despertando interesse em outros pesquisadores ao longo dos últimos anos e o campo do conhecimento vem se desenvolvendo nesse sentido. O cluster formado pelos autores com publicações mais recentes tem como autor mais relevante Gerlak, A.K. e possui 10 outros autores, demonstrando o aumento crescente de trabalhos no tema. Estes últimos se mostram, pela análise da proximidade dos clusters, mais complementares à obra de Varady et al.

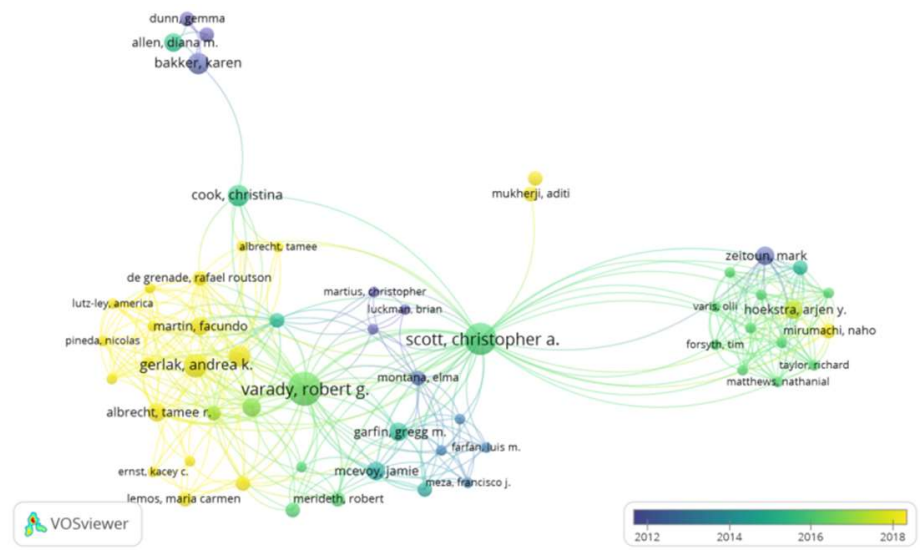

Figura 6: Mapa bibliométrico de coautoria, por autores (overlay visualization). Fonte: Web of Science (2021).

Van Eck et al. (2014) relata que a relação de coocorrência entre duas palavras-chave é definida pelo número de publicações em uma base de documentos em que ambas ocorrem conjuntamente, seja no título, no resumo ou na lista de palavras-chave. Na composição do seguinte mapa de palavras-chave (Figura 7) foi utilizado como critério de elaboração a seleção de termos com, no mínimo, dez menções nos 388 títulos considerados pela pesquisa. Dos 1.866 termos que foram inicialmente considerados pelo software, 56 itens constam no gráfico pelo seu grau de significância.

A figura 7 demonstra a existência de cinco conglomerados de termos. 0 cluster 1 , no qual a expressão climate-change (mudanças climáticas) figura em destaque com 82 ocorrências, aparece em vermelho e nele constam 55 links relacionados e força equivalente a 324. Destacam-se governance 
(governança), challenge (desafio), policy (política) e science. (ciência). O cluster 2, retratado na cor verde, traz a expressão water security (segurança hídrica), que é o termo de maior destaque entre as produções científicas analisadas, despontando 167 vezes, com força de 522 e 54 links de co-ocorrência entre termos. Quality (qualidade), vulnerability (vulnerabilidade) e impact (impacto) também são palavras que merecem destaque. 0 cluster 3, em azul, tem sua estruturação centrada da palavra Management (gestão) que tem 77 ocorrências. Nota-se que outras expressões como water resources (recursos hídricos), drough (seca) e groundwater (águas subterrâneas) possuem grande frequência de utilização.

O cluster 4, retratado em amarelo, foi estruturado em torno das expressões scarcity (escassez) com 31 ocorrências - e, novamente, climate change (mudanças climáticas), dessa vez utilizada sem o hífen e com 39 ocorrências. Destaca-se ainda sustainability (sustentabilidade) que ocorreu 24 vezes na análise. 0 quinto cluster, na cor roxa, tem como principal termo resources (recursos), com 51 ocorrências.

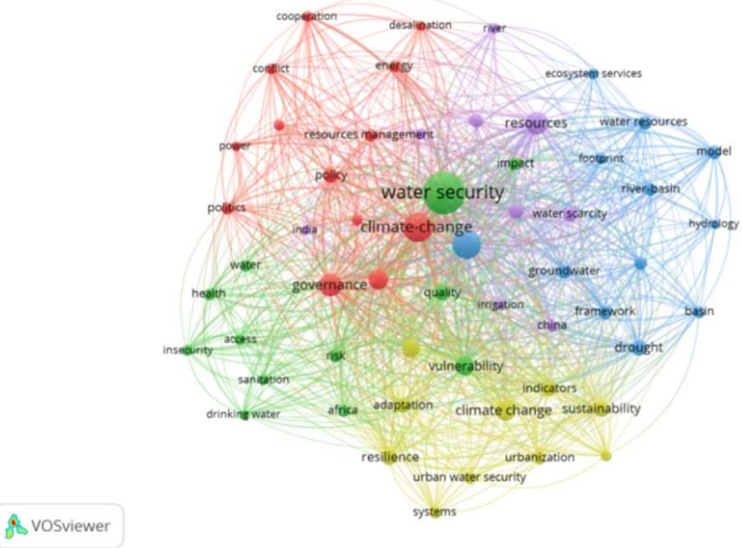

Figura 7: Rede de coocorrências de palavras-chave. Fonte: Web of Science (2021).

Na Figura 8 está representada a análise de frequência de coocorrência de palavras-chave quanto ao período. Depreende-se da análise do gráfico que as primeiras pesquisas sobre segurança hídrica estavam mais ligadas às mudanças climáticas, resiliência, acesso à agua e governança. Atualmente, percebe-se que o maior foco está nas questões de sustentabilidade, urbanização, segurança hídrica urbana, indicadores e aspectos do desenvolvimento sustentável. Além destes, destacam-se nos trabalhos atuais terminologias como saúde, saneamento e água potável. De forma geral, o panorama apresentado graficamente pelo software em utilização mostra uma intensa conexão entre os termos apresentados, de modo que estão todos de alguma forma inter-relacionados.

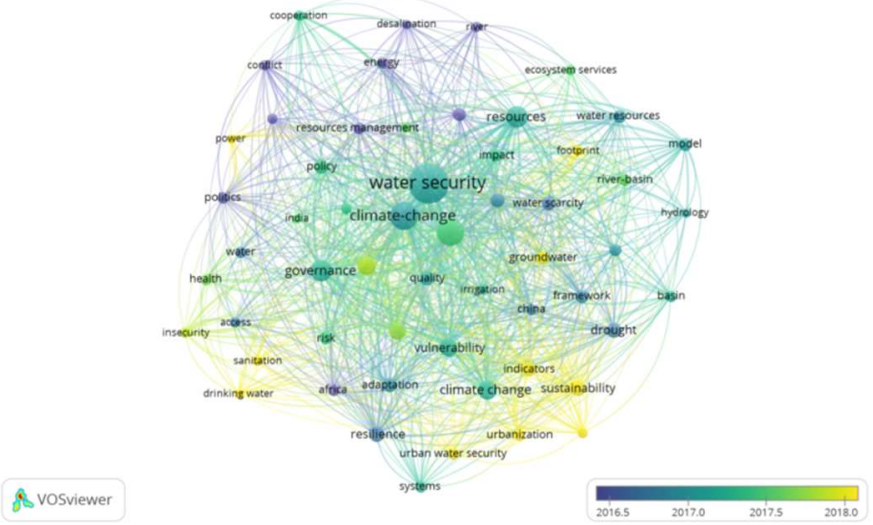

Figura 8: Coocorrências de palavras-chave (overlay visualization). Fonte: Web of Science (2021). 


\section{CONCLUSÕES}

Considerando a base dados da Web of Science, este artigo objetivou analisar a produção de pesquisa internacional sobre a segurança hídrica em um recorte temporal de 2010 a 2021. Dentro do tema selecionado, foram consideradas 388 publicações em vários aspectos, tais como como o número de documentos disponíveis, países com mais produções, periódicos onde foram feitas as publicações, autores mais notáveis e palavras-chave. Executou-se um estudo bibliométrico no qual foram utilizadas técnicas avaliativas e relacionais. As principais conclusões são demonstradas a seguir: a) A temática da segurança hídrica experimentou um rápido desenvolvimento científico desde 2015. Esse cenário ressalta a importância que o tema vem ganhando nos últimos anos, tanto no cenário acadêmico, quanto político, econômico, social, da saúde pública etc. A análise feita permitiu observar que os maiores expoentes da produção científica nessa temática estão em instituições concentradas em países como Estados Unidos, China, Canadá, Inglaterra, Austrália, Holanda, Alemanha, Índia, Suécia e África do Sul. Os três periódicos mais influentes são: Water, Current Opinion in Environmental Sustainability e Science of the Total Environment; b) A publicação de maior impacto foi publicada em 2010 e intitulada "Global threats to human water security and river biodiversity", escrita por Voeroesmarty, C.; McIntyre, P. B.; Gessner, M. O. et. al.; c) No que tange à coocorrência de palavras-chave quanto ao período, observa-se que atualmente há uma maior preocupação com os aspectos de vulnerabilidade social, urbanismo e desenvolvimento sustentável relacionado à segurança hídrica, ampliando a gama de abordagens sobre o tema, além do surgimento de pesquisas sobre segurança hídrica urbana; d) Importante ressaltar que a pesquisa ora realizada teve como única base de dados consultada a Web of Science, considerada uma das mais expressivas no meio científico. Ainda assim, coloca-se que não houve esgotamento no desenvolvimento do tema, pois outras bases relevantes podem ser consideradas, a exemplo da Scopus, Scielo, Google Scholar, EBSCO, além de periódicos específicos da área. Além disso, podem ser utilizados outros índices de pesquisa ou delimitações temporais na mesma base de dados. Essa é, inclusive, uma sugestão para elaboração de outros trabalhos.

De maneira geral, as conclusões deste estudo visam oferecer uma visão abrangente e sistemática do panorama geral do avanço das publicações da última década na temática da segurança hídrica, com o objetivo de compreender melhor como se deu a evolução científica nessa temática e podendo servir de referência para pesquisas futuras.

\section{REFERÊNCIAS}

BOGARDI, J. J.; DUDGEON, D.; LAWFORD, R.; FLINKERBUSCH E.; MEYN, A.; PAHL-WOSTL, C.; VIELHAUER, K.; VOROSMARTY, C.. Water security for a planet under pressure: interconnected challenges of a changing world call for sustainable solutions. Current Opinion in Environmental Sustainability, v.4, n.1, p.35-43, 2012. DOI: https://doi.org/10.1016/j.cosust.2011.12.002

COATES, V.; FAROOQUE, M.; KLAVANS, R.; LAPID, K.; LINSTONE, H. A.; PISTORIUS, C.; PORTER, A. L.. On the Future of Technological Forecasting. Elsevier Science, v.67, n.1, p.
1-17, 2001. DOI: https://doi.org/10.1016/S00401625(00)00122-0

COOK, C.; BAKKER, K.. Water security: debating an emerging paradigm. Global Environmental Change, v.22, p.94-102, 2012. DOI: https://doi.org/10.1016/j.gloenvcha.2011.10.011

COSGROVE, C. E.; COSGROVE, W. J.. The dynamics of global water futures driving forces 2011-2050. Global Water Futures 2050. United Nations Educational, Scientific and Cultural Organization. Paris, 2012. 
GRÁCIO, M. C. C.. Acoplamento bibliográfico e análise de cocitação: revisão teórico-conceitual. Revista Eletrônica de Biblioteconomia e Ciência da Informação, v.21, n.47, p.82, 2016. DOI: https://doi.org/10.5007/15182924.2016v21n47p82

GREY, D.; GARRICK, D.; BLACKMORE, D.; KELMAN, J.; MULLER, M.; SADOFF, C.. Water security in one blue planet: twenty-first century policy challenges for science. Philosophical Transactions of the Royal Society A, v. 371, 2013. DOI: https://doi.org/10.1098/rsta.2012.0406

GREY, D.; SADOFF, C. W.. Sink or swim? Water security for growth and development. Water Policy, v.9, p.545-571, 2007. DOI: https://doi.org/10.2166/wp.2007.021

HOEKSTRA, A. Y.; BUURMAN, J.; VAN GINKEL, K. C. H.. Urban water security: a review. Environmental Research Letters, v.13, n.5, 2018. DOI: https://doi.org/10.1088/17489326/aaba52

MEKONNEN, M. M.; HOEKSTRA, A. Y.. Four billion people facing severe water scarcity. Science Advances, v.2, 2016. DOI: https://doi.org/10.1126/sciadv.1500323
PINK, R.. Child rights, right to water and sanitation, and human security. Health and Human Rights Journal, v.14, n.1, p.78-87, 2012

PNUD. Programa Das Nações Unidas Para O Desenvolvimento. Objetivos do Desenvolvimento Sustentável. Nova York: PNUD, 2015.

UN WATER.. Water Security \& the Global Water Agenda A UN-Water Analytical Brief. Hamilton: UNU-INWEH, 2013.

UNGA. United Nations General Assembly. Human Right to Water and Sanitation. Geneva: UNGA, 2010

VAN ECK, N. J.; WALTMAN, L.; DEKKER, R.; VAN DEN BERG, J. CitNetExplorer: a new software tool for analyzing and visualizing citation networks. Journal of Informetrics, v.8, n.4, p.802-823, 2014. DOI: https://doi.org/10.1016/j.joi.2014.07.006

WHEATER, H. S. P.. Water security and the science agenda. Water Resources Research, v.51, n.1, p.5406-5424, 2015. DOI: https://doi.org/10.1002/2015WR016892 